\title{
Development of an Optical Fiber Sensor Interrogation System for Vibration Analysis
}

\author{
Alfredo Lamberti, ${ }^{1}$ Ben De Pauw, ${ }^{1,2}$ and Steve Vanlanduit ${ }^{1,3}$ \\ ${ }^{1}$ Department of Mechanical Engineering, Vrije Universiteit Brussel, Pleinlaan 2, 1050 Brussels, Belgium \\ ${ }^{2}$ Department of Applied Physics and Photonics, Vrije Universiteit Brussel, Pleinlaan 2, 1050 Brussels, Belgium \\ ${ }^{3}$ Op3Mech Research Group, Faculty of Applied Engineering, University of Antwerp, Salesianenlaan 90, 2660 Antwerp, Belgium
}

Correspondence should be addressed to Steve Vanlanduit; steve.vanlanduit@uantwerpen.be

Received 21 November 2015; Accepted 7 March 2016

Academic Editor: Lun-Kai Cheng

Copyright (C) 2016 Alfredo Lamberti et al. This is an open access article distributed under the Creative Commons Attribution License, which permits unrestricted use, distribution, and reproduction in any medium, provided the original work is properly cited.

\begin{abstract}
Since the introduction of dynamic optical fiber sensor interrogation systems on the market it has become possible to perform vibration measurements at frequencies up to a few $\mathrm{kHz}$. Nevertheless, the use of these sensors in vibration analysis has not become a standard practice yet. This is mainly caused by the fact that interrogators are stand-alone systems which focus on strain measurements while other types of signals are also required for vibration analysis (e.g., force signals). In this paper, we present a fiber Bragg grating (FBG) interrogation system that enables accurate strain measurement simultaneously with other signals (e.g., excitation forces). The system is based on a Vertical Cavity Surface Emitting Laser (VCSEL) and can easily be assembled with relatively low-cost off-the-shelf components. Dynamic measurements up to a few tens of $\mathrm{kHz}$ with a dynamic precision of around 3 nanostrain per square-root $\mathrm{Hz}$ can be performed. We evaluate the proposed system on two measurement examples: a steel beam with FBG sensors glued on top and a composite test specimen with a fiber sensor integrated within the material. We show that in the latter case the results of the interrogation system are superior in quality compared to a state-of-the-art commercially available interrogation system.
\end{abstract}

\section{Introduction}

A fiber Bragg grating (FBG) consists of a periodic refractive index change over a certain length of an optical fiber. When a broadband light source is coupled into the fiber, light at a narrow wavelength band is reflected by the grating. This wavelength is depending, amongst others, on the pitch of the grating. When a dynamic strain is applied to the grating a shift in the reflected wavelength can be observed (the strain sensitivity of typical FBG sensors is $1.2 \mathrm{pm} / \mu \epsilon$ ). Several interrogation mechanisms exist to read out the reflected so-called Bragg wavelength peak [1-3]. Most commercially available fiber Bragg grating interrogation systems use a broadband light source in combination with a full-spectrum measurement of the reflected and/or transmitted light spectrum (this full-spectrum measurement is in particular necessary in the case of multiplexed sensors). The rate of the readout is limited by the scan speed of the spectral measurement technique. Recently, MEMS based tunable filter technologies have been proposed to measure the reflected spectrum with a sample rate up to $100 \mathrm{kHz}$ [4]. While this method has a large potential in the framework of vibration analysis, the used components are not yet commercially available.

A second class of techniques uses a tunable laser instead of a tunable detector. External cavity semiconductor tunable lasers that can be used for this purpose are however of very high cost. Vertical Cavity Surface Emitting Lasers (VCSELs) are an attractive alternative that can be used to realize a low cost tunable laser. The working principle of these VCSELs is simple: they produce a narrowband laser of which the wavelength can be tuned by changing the driving current [5]. Recent work has shown that these VCSELs can be used to successfully realize an accurate FBG interrogator system [6]. Because of the low power requirement and the compact size of a VCSEL, miniature VCSEL based interrogators can even be manufactured [7]. In $[8,9]$ the authors showed that by using VCSELs dynamic measurements with a dynamic range of $30 \mathrm{~dB}$ can be performed. 


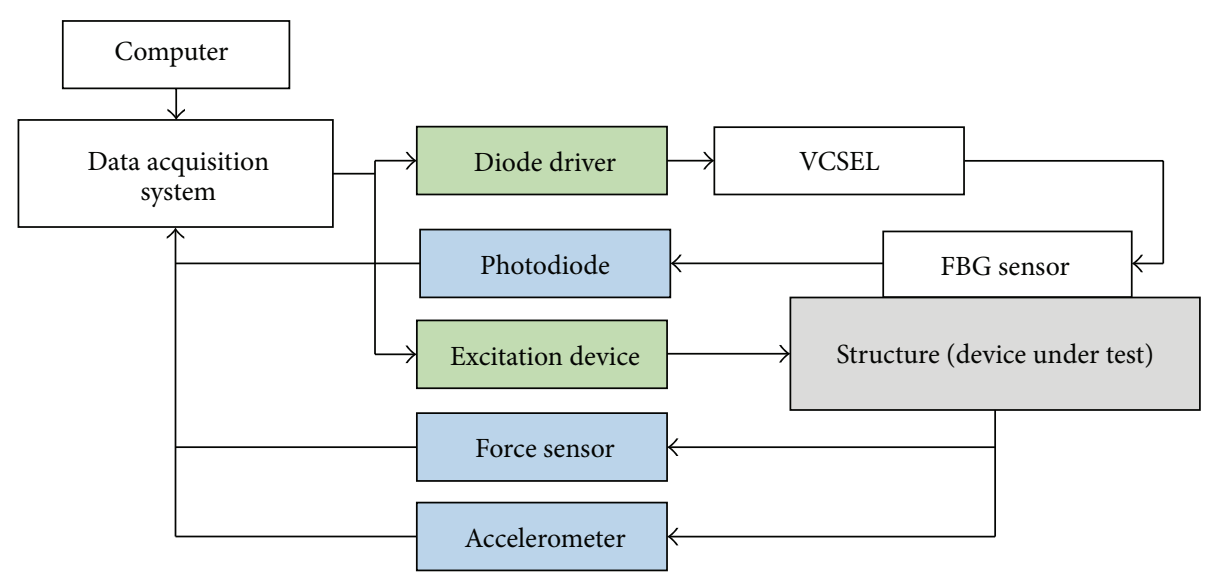

FIGURE 1: Layout of the proposed VCSEL based FBG interrogation system. The experiment is fully controlled by the multipurpose data acquisition system.

In this paper we propose a VCSEL based measurement system that can be used for vibration analysis. More specifically, it will be shown that the proposed system has the following specifications:

(i) The system allows the user to perform optical fiber based strain measurements simultaneously with other measurement quantities (accelerometers, forces, etc.). This is essential when performing vibration analysis, as, for instance, an experimental modal analysis [10].

(ii) All components in the system can be purchased offthe-shelve and the construction of the setup does not require advanced expertise in electronics or optics.

(iii) The system results in fairly high resolution full-spectrum measurements at sample rates of a few tens of $\mathrm{kHz}$.

(iv) The dynamic precision is around $3 \mathrm{n} \epsilon$ per square root $\mathrm{Hz}$, which is comparable with other commercial interrogators.

(v) The interrogator is robust with respect to distortions in the optical spectrum (which are typically caused when integrating a FBG sensor in a composite material).

In Section 2 the system is described in detail. In Section 3 we illustrate the performance of the system on two test cases:

(i) A vibration measurement of a steel beam with an optical fiber glued on top.

(ii) A vibration measurement of a composite T-joint specimen with an integrated optical fiber.

Currently, there are many FBG peak wavelength interrogators on the market which operate on a different measurement strategy or peak detection scheme. In this paper we will compare the measurement results of the proposed tests system to the results of a commercially available interrogator: the FBG-scan 700 interrogator from the company FBGS. We will illustrate that the developed VCSEL based interrogator gives equal measurement results compared to the FBG-scan
700 for the first test case. For the second test case, in which peak distortions occur because of the integration, we will show that the performance of our VCSEL based interrogator is superior than the used commercial interrogator. This can be explained by the fact that a higher wavelength resolution is obtained in our interrogator and that a more robust peak detection method is used [11].

\section{Materials and Methods}

2.1. The Proposed Measurement System. Our proposed FBG interrogation system is built around a (commercially available) multifunction data acquisition (DAQ) board which is controlled by a personal computer. The setup is shown in Figure 1. The analog outputs channels of the DAQ board are used to generate two signals (see green blocks in Figure 1):

(i) A sawtooth signal that is used to generate a linear shift in the wavelength that is produced by the VCSELs.

(ii) A (periodic) excitation signal which is used to drive the excitation device to generate controlled vibrations in the structure.

Two analog input channels are used to measure two signals: (a) the optical power of the VCSEL signal that is reflected by the FBG sensor and (b) force transducer and accelerometer sensor signals. Because these signals are measured by the same DAQ device the measurements can be obtained simultaneously.

In this paper we have used the following system components:

(i) Computer: a Dell Latitude E5440 laptop (the measurements are performed, processed, and visualized in Matlab).

(ii) Data acquisition system: a National Instruments NIUSB-6363 DAQ device (the device has a maximum sample rate of $1 \mathrm{MHz}$ (when two analog inputs are measured). This sample rate determines the scan rate of the interrogator system: when using, for example, 


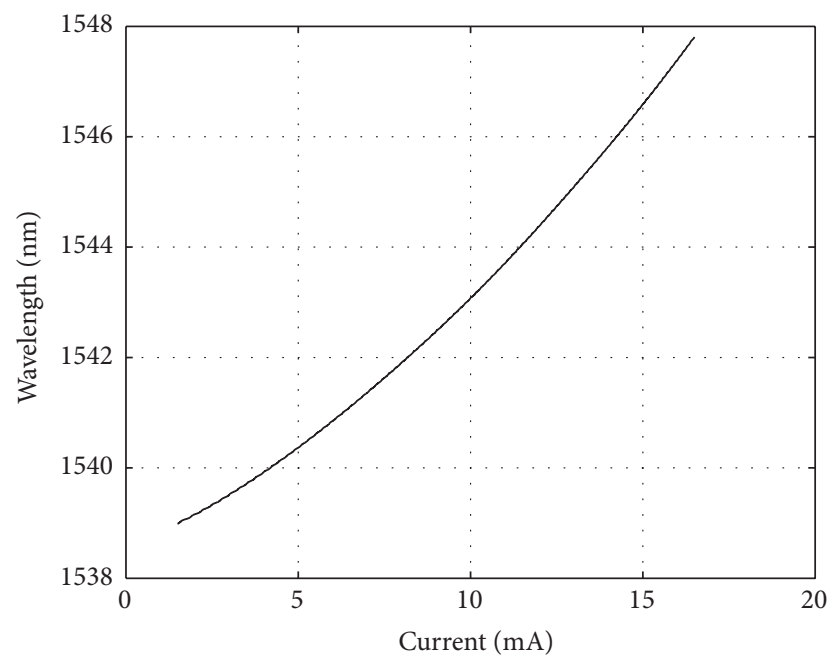

FIGURE 2: Center wavelength of the VCSEL signal in function of driving current.

an interrogation of 100 wavelengths, full-spectrum measurements can be performed at a rate of $10 \mathrm{kHz}$. This principle will be explained in more detail in Section 2.2).

(iii) Diode driver: Thorlabs type LDC 200C Diode driver.

(iv) VCSELs: VTEC $1550 \mathrm{~nm}$ pigtailed single mode VCSEL with an output power of $0.5 \mathrm{~mW}$.

(v) Photodiode: Hamamatsu InGaAs Photodiode (A Thorlabs type PDA200C amplifier is used to measure the current signals. The amplifier has six gain factors. In the paper the $0.1 \mathrm{mV} / \mathrm{V}$ range was used (with this range the measurement bandwidth is $250 \mathrm{kHz}$ )).

(vi) FBG sensors: FBGS draw tower gratings.

(vii) Excitation device: Bruel \& Kjaer Type 4810 electrodynamic shaker.

(viii) Force sensor and accelerometer: PCB piezoelectric transducers.

(ix) Device under test: (1) steel beam and (2) carbon fiber composite T-joint specimen.

The diode driver generates a current of $2 \mathrm{~mA}$ through the VCSEL for each volt that is applied to the driver (the input voltage is generated by the DAQ board signal generator). By applying a linear voltage signal between $0.75 \mathrm{~V}$ and $8.25 \mathrm{~V}$ one can thus sweep the current between $1.5 \mathrm{~mA}$ and $16.5 \mathrm{~mA}$. This results in a sweep of the wavelength of the VCSEL between $1538 \mathrm{~nm}$ and $1548 \mathrm{~nm}$ as can be seen in Figure 2. The wavelength sweep is not perfectly linear over the complete range, but in the context of vibration measurements this does not pose any problem because the wavelength shift due to vibrations will be limited to a few picometers only.

In order to perform a measurement in a certain wavelength range $\left[\lambda_{\min }, \lambda_{\max }\right]$, the VCSEL is driven using a sawtooth signal which linearly varies between two currents that correspond to the lower wavelength $\lambda_{\min }$ and upper wavelength $\lambda_{\max }$ (using the current conversion factors shown

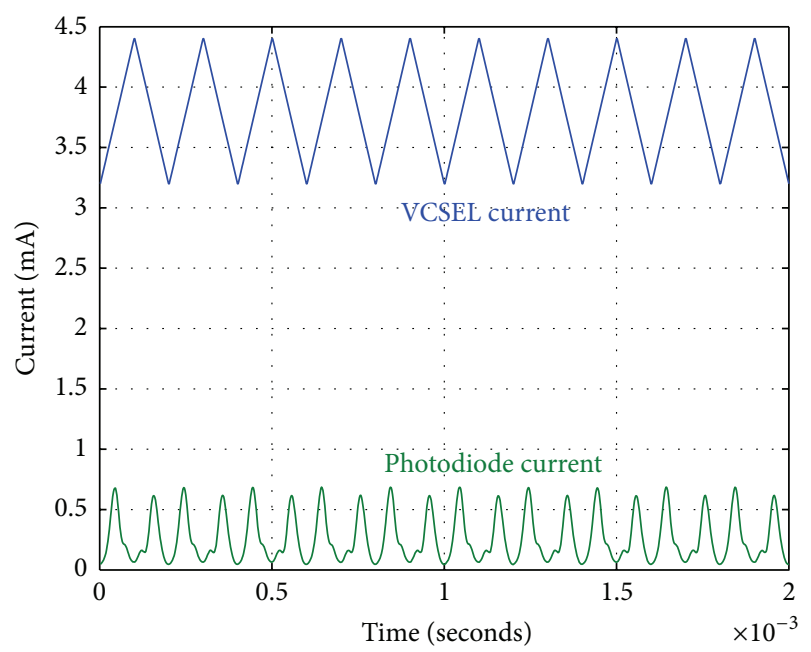

FIGURE 3: Illustration of the measurement principle. The blue line represents the VCSEL current and the green curve corresponds to the photodiode current of the signal reflected from the FBG sensor. For each sawtooth period two Bragg peaks are visible.

in Figure 2). The photodiode measures the spectrum of the reflected signal power in the wavelength band $\left[\lambda_{\min }, \lambda_{\max }\right]$. This Bragg peak measurement principle is illustrated in Figure 3. From Figure 3 it can be seen that a Bragg peak in the photodiode current (see green line) is observed twice for each period of the sawtooth shaped VCSEL current signal (blue line in Figure 3): both at the rising edge of the sawtooth and at the falling edge of the sawtooth a peak occurs in the VCSEL signal. In the remainder of the paper we will use only the Bragg peak of the rising edge.

The vector containing the measured photodiode currents (see green curve in Figure 3) can be converted to wavelengths by using the conversion curve given in Figure 2. The resulting vector can be transformed into a matrix by putting every record corresponding to one rise and fall of the sawtooth in one column. In this matrix the evolution of the Bragg peak in function of time is visible as can be seen in Figure 4. In the next paragraph we will describe how we obtain the quantitative wavelength shift values in function of time.

2.2. Data Processing. The objective of the proposed data processing technique is to obtain the peak location in function of time in the matrix given in Figure 4. Classical interrogation methods use quadratic or cubic interpolation of the peak to detect the maximum with subwavelength resolution. It is known that this method can result in inaccurate results if the Bragg peak is distorted [11]. In this paper we will use the phase correlation method that was proposed recently in [12]. The method consists of the following steps:

(1) Calculate the FFT of the different columns of the data matrix A represented in Figure 4 to obtain the complex matrix $\mathbf{B}$.

(2) Calculate the phase value of each element of the complex valued FFT matrix $\mathbf{B}$ (using the Matlab command angle(B)). Denote the resulting matrix $\boldsymbol{\theta}_{\mathbf{B}}$. 


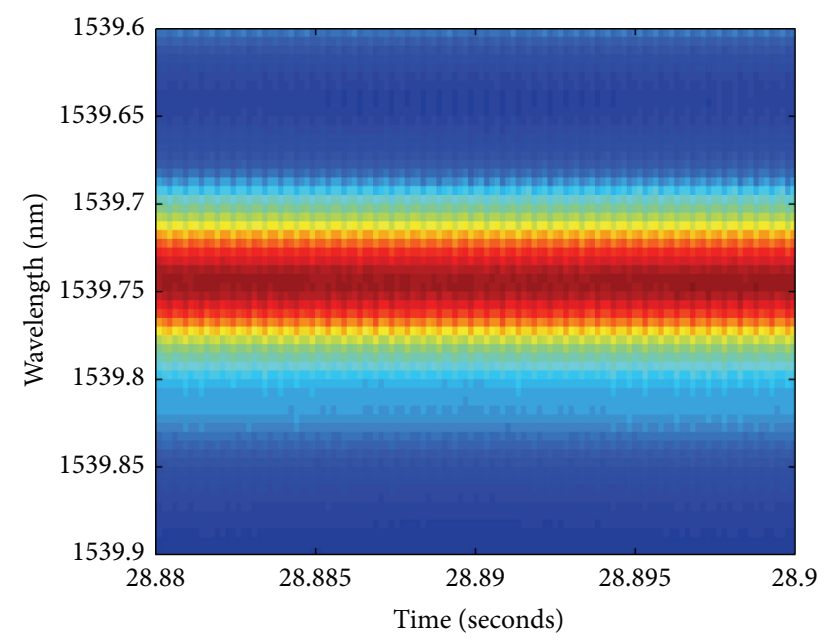

FIgURE 4: Measurement data matrix (denoted by $\mathbf{A}$ ) resulting by stacking the wavelength spectrum measured at time $t_{i}(i=$ $1, \ldots, n)$ in column $i$. A zoom of $20 \mu \mathrm{sec}$ was made in order to be able to illustrate the oscillations of the Bragg peaks (the complete measurement period that was measured has a duration of 40 seconds).

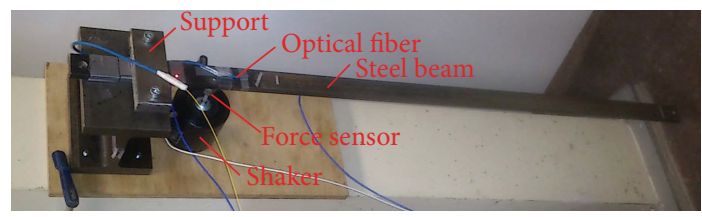

FIgURE 5: Setup of the steel beam experiment.

(3) Scale the matrix $\boldsymbol{\theta}_{\mathrm{B}}$ by multiplying each element in row $k$ of the matrix by $N \lambda_{0} / 2 \pi k$ to obtain the matrix $\Delta_{\lambda}$ (where $\lambda_{0}$ is the wavelength resolution of the measurement).

(4) Calculate the median value of the matrix $\Delta_{\lambda}$ along the columns to arrive at the vector containing wavelength shift in function of time: $\delta \lambda\left(t_{1}, \ldots, t_{n}\right)$ (with $n$ the number of columns of $\mathbf{A}$ ).

In this paper we have applied the above-mentioned phase correlation method to obtain strain values from all VCSEL based interrogator measurements. The subwavelength shifts (and hence strains) from the FBG-scan spectra shown in the remainder of the paper were obtained from the maximum of a quadratic peak fit (this is the peak detection method that is used in the software provided by the manufacturer FBGS). In the next section we will illustrate the measurement system on two vibration analysis test cases: a cantilevered steel beam and a cantilevered carbon fiber composite test sample.

\section{Results and Discussion}

3.1. Steel Beam Test Case. In the first example, a cantilever steel beam was tested. The steel beam was clamped on a steel I-profile. An electrodynamic shaker was fixed below the beam at a position of $10 \mathrm{~cm}$ from the clamping (see Figure 5). A force transducer was used to measure the excitation force generated by the shaker. The beam was instrumented with a single mode optical fiber in which 22 FBG sensors were placed (distributed equally along the length of the beam).

The FBG sensors were read out sequentially with the FBG-scan 700 interrogator (from company FBGS) and with the proposed VCSEL based interrogator. The optical spectra of both interrogators and their standard deviation (obtained from 10 independent measurements) are shown in Figure 6. From the figure it can be observed that the SNR of the FBGscan is around $50 \mathrm{~dB}$ compared to about $35 \mathrm{~dB}$ for the VCSEL based system. This can be explained by the fact that the optical power of the light source of the FBG-scan interrogator is considerably higher than that of the VCSEL (the maximum power of the VCSEL is only $0.5 \mathrm{~mW}$ ). Also, the bandwidth of the FBG-scan interrogator is significantly larger: $40 \mathrm{~nm}$ compared to $3 \mathrm{~nm}$ for the VCSEL based interrogator (it can be noted from Figure 2 that the wavelength bandwidth of the VCSEL can go up to $9 \mathrm{~nm}$, but we have limited ourselves to a smaller bandwidth in in which the dependency between current and wavelength is linear). This means that in the VCSEL based interrogator only four out of twenty-two gratings can be measured (remark that this could be extended by using multiple VCSELs in the measurement system). It should be noted the bandwidth and power of the used VCSEL are somewhat limited compared to the used commercial FBG interrogator. However, both the power and bandwidth of commercially available VCSELs are continuously increasing (the tests performed in the paper were performed in May 2015 with a $0.5 \mathrm{~mW}$ VCSEL and six months later $2 \mathrm{~mW}$ VCSELs are available on the market).

In the current experiment we aim at reading out the FBG sensors with the VCSEL based system at a rate of $5 \mathrm{kHz}$ (compared to the $2 \mathrm{kHz}$ maximum readout of the FBG-scan system). Because we have used a sample rate of the DAQ system of $500 \mathrm{kHz}$ (this is the maximum sample rate for four input channels for our device) this means that we have 100 wavelengths in each measurement, giving a wavelength resolution of $30 \mathrm{pm}$ (compared to $80 \mathrm{pm}$ for the FBG-scan system). We could reduce this resolution by increasing the sample rate (up to $1 \mathrm{MHz}$ is possible with the current device in case two inputs are acquired) or by decreasing the rate of the readout. In principle it is possible to read out FBG sensors at higher rates than the $5 \mathrm{kHz}$ rate used in the paper. One should be careful when using high frequency sawtooth signals to drive the VCSEL because of the following two reasons:

(i) The laser diode driver has a limited low-pass frequency (in our case the Thorlabs type LDC 200C has a maximum low-pass frequency of $250 \mathrm{kHz}$ ). This means that at high frequencies the sawtooth will be low-pass filtered.

(ii) The VCSEL introduces hysteresis at high frequencies.

In order to test and compare the dynamic response of both interrogator systems we have used two types of excitation signals:

(i) Sinusoidal excitation at $182 \mathrm{~Hz}$ (near the third resonance frequency of the beam). 


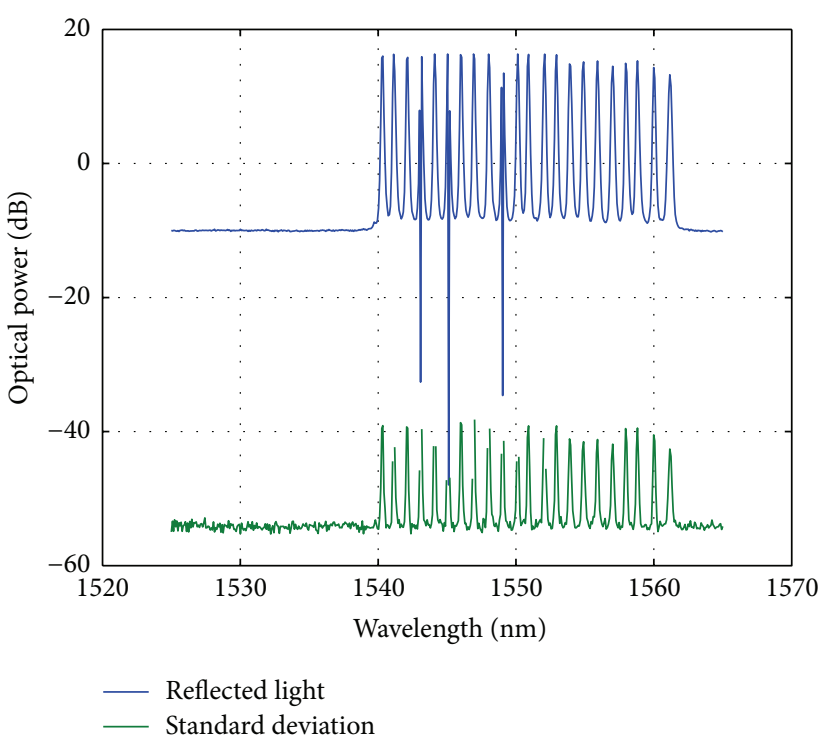

(a)

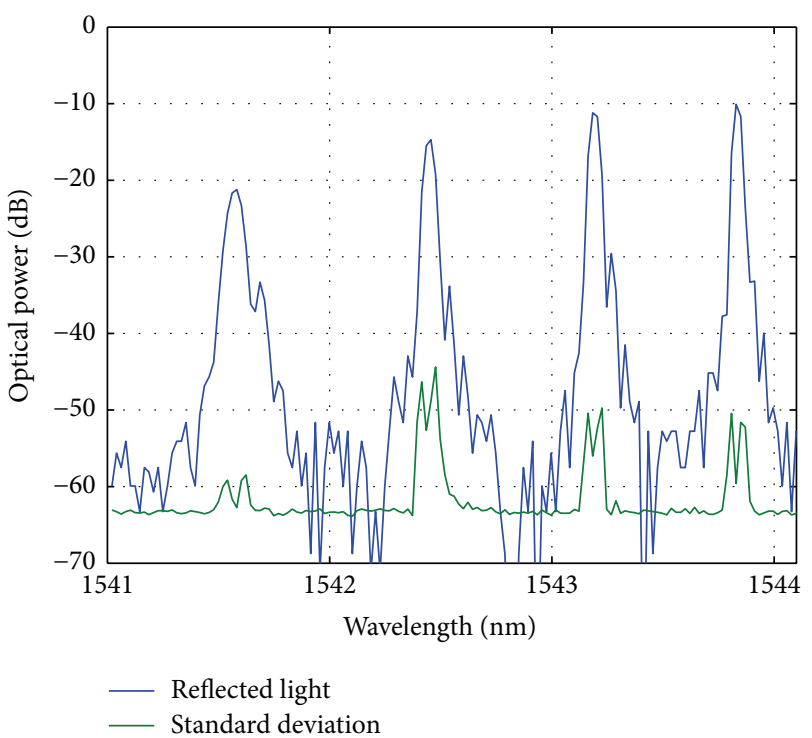

(b)

FIGURE 6: Optical spectrum and standard deviation. (a) FBG-scan 700 interrogator, (b) VCSEL based interrogator. It can be observed that the SNR of the FBG-scan system is about $15 \mathrm{~dB}$ larger, but on the other hand the wavelength resolution of the VCSEL based interrogator is 2.7 times higher. Note that the FBG spectra contain a sharp power drop-off at three of the twenty-two peaks. This is caused by the saturation due to an integration time which was set too high. These three peaks were not used in the analysis presented in the paper.

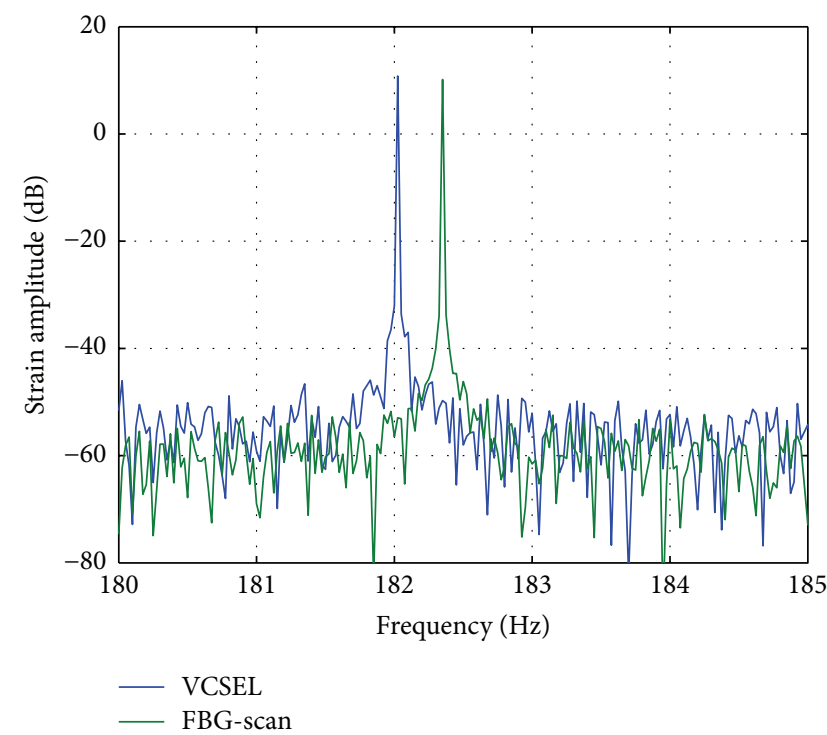

(a)

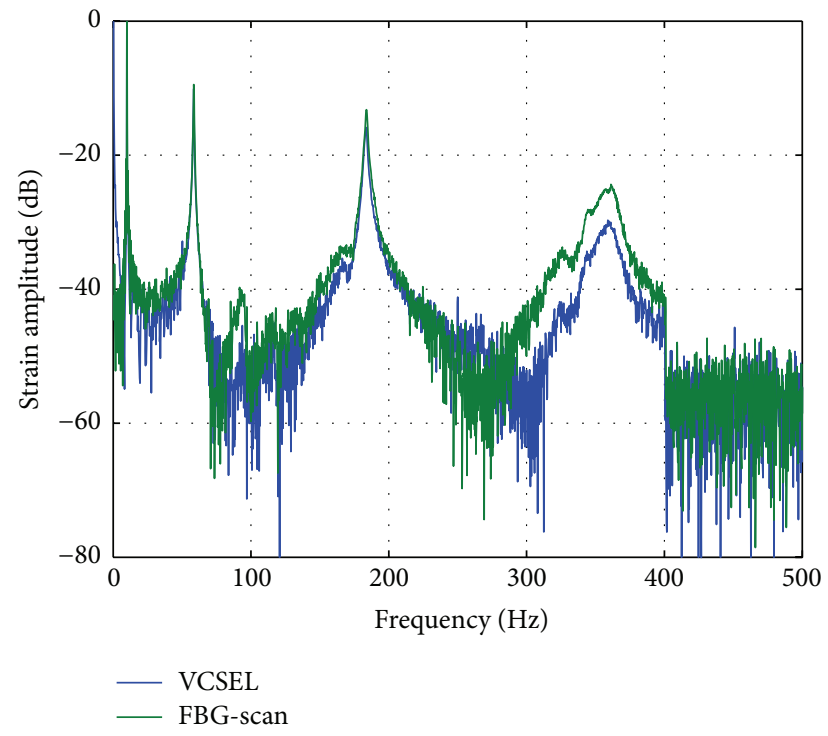

(b)

FIGURE 7: Measured vibration spectra: (a) when using sinusoidal excitation, (b) when using swept sine excitation.

(ii) Periodic chirp excitation between $0.25 \mathrm{~Hz}$ and $500 \mathrm{~Hz}$.

The results of these measurements using both interrogation systems are shown in Figure 7.

From Figure 7(a) it can be concluded that the SNR of the vibration measurements is the same for both measurement systems: about $60 \mathrm{~dB}$. The noise floor of the measurements is at $-50 \mathrm{~dB}$ which gives us a strain level precision of $3 \mathrm{n} \epsilon$ (the $0 \mathrm{~dB}$ reference in the plot is chosen equal to $1 \mu \epsilon$ ). The measured amplitude of the sinusoid obtained using both systems is equal (the difference is about $1 \mathrm{~dB}$ ), but it can be observed that the frequency of the FBG-scan interrogator does not perfectly correspond with the excitation frequency. This is caused by the fact that the sample times of the FBGscan system are not synchronized with the excitation. It should be noted that several commercial interrogators allow synchronization with external signals but this option was not used in the paper. Because of this lack of synchronization it is not straightforward to calculate the Frequency Response Functions (FRFs) using the FBG-scan system (the FRF is defined by the Fourier spectrum of the strain divided by 


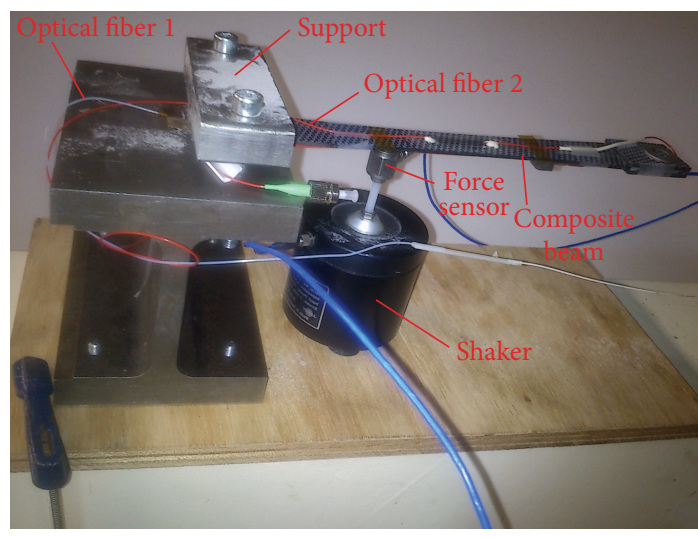

FIGURE 8: Setup of the composite beam experiment.

the Fourier spectrum of the excitation force applied to the structure with the shaker). Since the VCSEL based interrogator and the shaker are controlled by the same DAQ board, the measurements of the FBG sensors and the force are obtained simultaneously (and hence they are automatically synchronized).

From the response of the periodic chirp (see Figure 7(b)) the first four resonance frequencies of the beam can be identified. The vibration spectra corresponding to both measurement systems match very well (note that the strain levels are very low, below $0.1 \mu \epsilon$ ).

From the experiment we can conclude that the VCSEL based interrogator enables us to obtain measurements with a precision that is close to the one of the commercially available interrogator (FBG-scan 700). With our VCSEL based system we can simultaneously measure strain (using the FBG sensor) and force signals. This allows us to more easily calculate Frequency Response Functions (FRFs).

In the next paragraph we will show that the proposed interrogator system is much more robust for distortions of the optical FBG spectra.

3.2. Composite Beam Test. In this section we give the results of vibration measurements on a carbon fiber T-joint specimen (see setup in Figure 8). The structure was instrumented with a fiber sensor (with one FBG grating) that was inserted in the middle of the specimen during the manufacturing process (remark that there is a second fiber sensor attached on the specimen but this one was not used in the test because the wavelength is outside the range of the interrogator). As was the case in the steel beam example, we used a shaker to excite the beam and a force sensor to measure the excitation force. Again, the FBG sensor was read out with the FBG-scan 700 interrogator and the proposed VCSEL based system. The optical spectra (without any mechanical excitation applied) are shown in Figure 9.

The SNR values are similar to the ones measured in the steel beam case: $60 \mathrm{~dB}$ for the FBG-scan and $40 \mathrm{~dB}$ for the VCSEL based system. In this measurement case there are two important differences compared to the steel beam case which can be observed in Figure 9(b): (i) The spectral width of the Bragg peak is almost $500 \mathrm{pm}$ (compared to about $100 \mathrm{pm}$ in the case of the steel beam experiment).

(ii) The spectrum is heavily distorted: the top is not flat and side lobes appear.

This peak distortion, and more in particular peak broadening, is typical for sensors that are integrated in composite materials [13]. Note that because of the limited wavelength resolution in the FBG-scan analyzer these effects cannot be observed in Figure 9(a). In what follows we will show, however, that these effects can have a large influence on the vibration measurements. We note that in our peak detection algorithm we have assumed that the distortions are not changing during the loading of the specimen. Because in vibration engineering the strain variations are usually small this assumption is realistic (we have checked that there are indeed no significant changes of the peak distortions between the signals measured at different strain levels during the loading).

Ideally, one should compare the measured strains with a reference strain gauge. However, it is not possible to apply such a strain gauge internally in the composite material. Therefore, we have used two methods to validate our results: firstly, we evaluate the SNR of the two investigated interrogators when a pure sinusoidal excitation is applied. Secondly, we compare the resonance frequencies obtained from the FBG sensors with those obtained from an accelerometer (when applying a periodic chirp excitation). In the composite beam experiment the vibration spectra obtained using the FBGscan interrogator and the VCSEL based interrogator deviate significantly. Because of the distortions in the spectra, the signal-to-noise ratio (that can be observed when using the sinusoidal excitation) is about $12 \mathrm{~dB}$ lower when using the FBG-scan interrogator. Also, the broadband vibration spectrum of the FBG-scan system shown in green in Figure 10(b) is distorted (the resonance peak at $206 \mathrm{~Hz}$ cannot be identified anymore, while it is clearly present in the acceleration spectra). The proposed technique, on the other hand, is not sensitive to these distortions because of the following two reasons:

(i) The wavelength resolution is high enough to capture the distortion (16 pm compared to $80 \mathrm{pm}$ for the FBGscan).

(ii) The peak detection algorithm that was used (see Section 2.2) is robust for distortions in the spectra (as was shown in [11]).

Because we simultaneously measure force and acceleration signals with the VCSEL based interrogator we can also calculate Frequency Response Functions and compare the resulting FRFs for acceleration (from accelerometer) and strain (from FBG). The FRFs are displayed in Figure 11 and they were used as an input for a modal parameter estimation algorithm [14]. In Table 1 the results of the first four estimated modes from accelerometer and FBG sensor data are given. It can be seen that the errors on the identified resonance frequencies are very small (average relative error less than 


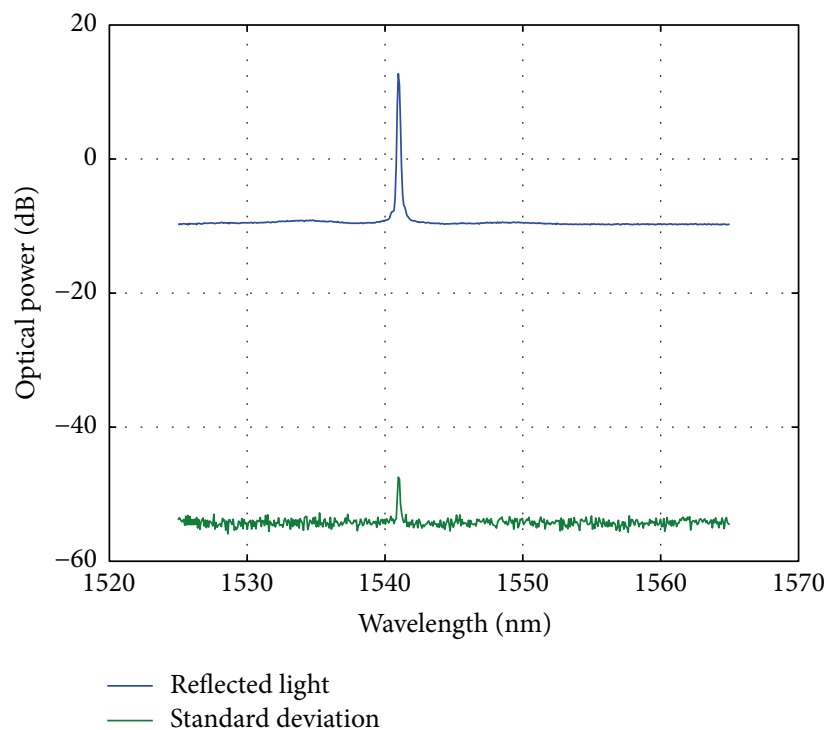

(a)

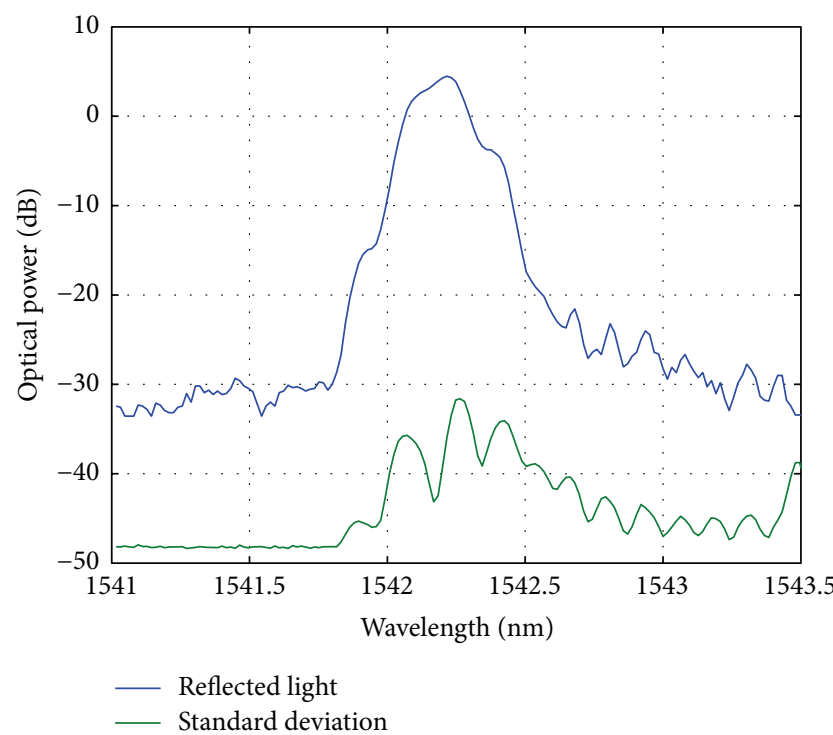

(b)

FIgURE 9: Optical spectrum and standard deviation. (a) FBG-scan 700 interrogator, (b) VCSEL based interrogator.

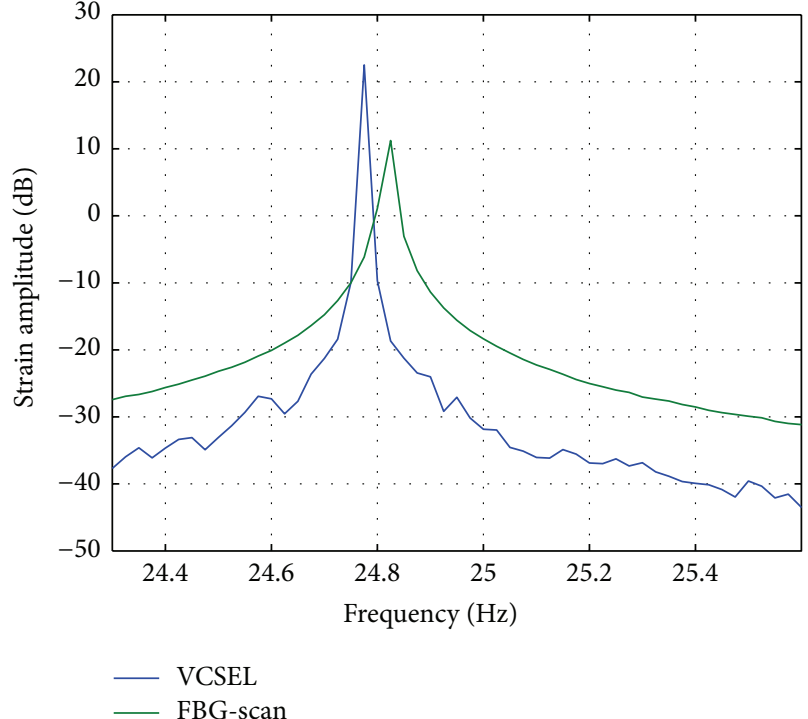

(a)

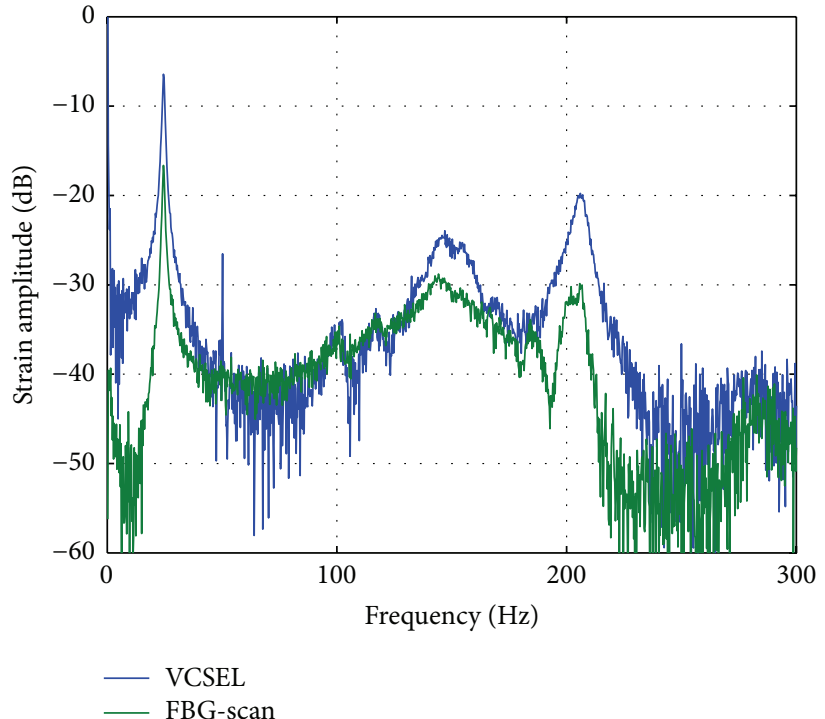

(b)

FIGURE 10: Measured vibration spectra: (a) when using sinusoidal excitation, (b) when using swept sine excitation.

$0.2 \%)$. The damping value estimates show an average relative deviation of $3 \%$ but in vibration analysis this is considered small as it is known that damping estimates usually have a significantly larger uncertainty.

From the results of the composite beam experiment we can conclude that the proposed system allows us to perform reliable vibration measurements even if the optical spectra are heavily distorted.

\section{Conclusions}

We have proposed an FBG sensor measurement system that is dedicated for vibration analysis. Because the system is completely controlled by a multifunction DAQ board it is possible to simultaneously acquire force and accelerometer measurements, and hence it is easy to calculate Frequency Response Functions. The hardware used in the proposed setup can be purchased off-the-shelf and it is fairly of low cost (the total cost of the system including the DAQ board is $4 k$ ). Using a vibration measurement of a steel beam we have shown that the proposed system results in measured vibration spectra that are comparable to the ones determined with a commercial interrogator. In case significant distortions are present in the optical spectra, the proposed system even outperforms traditional measurement systems.

Currently, the system has two limitations: (1) with current the optical power is limited to less than one $\mathrm{mW}$; (2) the bandwidth is limited to about $9 \mathrm{~nm}$. Ongoing technological 
TABLE 1: Estimated resonance frequencies and damping values.

\begin{tabular}{lcccccc}
\hline \multirow{2}{*}{ Mode } & \multicolumn{2}{c}{ Accelerometer } & \multicolumn{2}{c}{ FBG sensor } & \multicolumn{2}{c}{ Difference, in \% } \\
& $f_{n}^{\mathrm{acc}}$, in $\mathrm{Hz}$ & $\xi^{\mathrm{acc}}$, in $\%$ & $f_{n}^{\mathrm{FBG}}$, in $\mathrm{Hz}$ & $\xi^{\mathrm{FBG}}$, in $\%$ & $\left(f_{n}^{\mathrm{acc}}-f_{n}^{\mathrm{FBG}}\right) / f_{n}^{\mathrm{acc}}$ & 0 \\
$\left(\xi^{\mathrm{acc}}-\xi^{\mathrm{FBG}}\right) / \xi^{\mathrm{acc}}$ \\
\hline First bending & 22,7 & 1,9 & 22,7 & 1,8 & 0,6 & 5,3 \\
Second bending & 145,2 & 2,1 & 144,4 & 2,1 & 0 & 0 \\
First torsion & 206,5 & 1,5 & 206,6 & 1,4 & $-0,1$ & 6,7 \\
Third bending & 323,4 & 0,6 & 323,6 & 0,6 & 0 \\
\hline
\end{tabular}

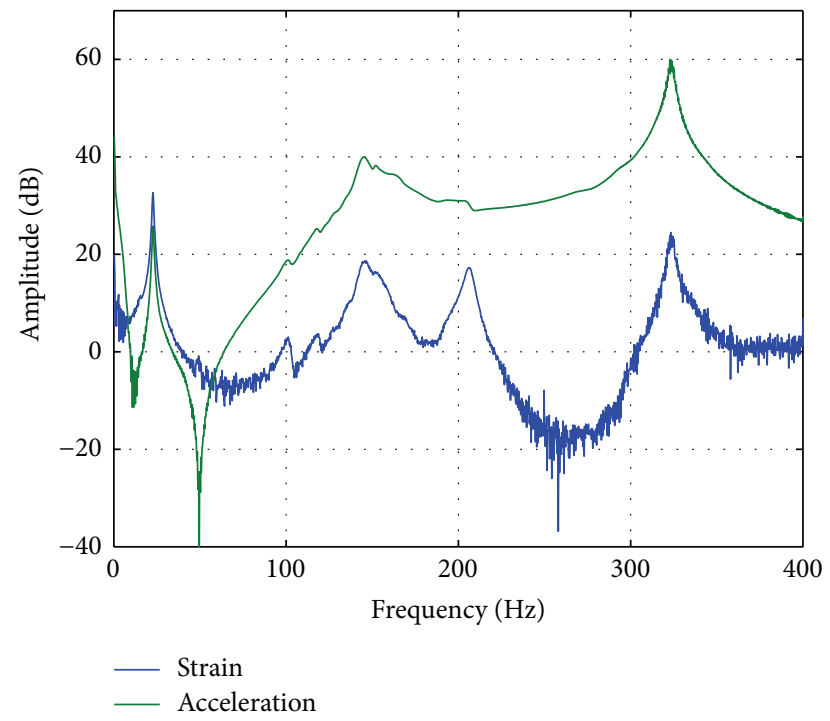

FIGURE 11: Measured Frequency Response Functions (FRFs) from acceleration (green dots) and FBG sensor (blue dots).

advances in VCSEL technologies, however, continuously extend the range of the available power and wavelength range.

\section{Competing Interests}

The authors declare that they have no competing interests.

\section{Acknowledgments}

This research has been sponsored by the Flemish Institute for the Improvement of the Scientific and Technological Research in Industry (IWT) in the framework of the SBO Project Self Sensing Composites. The authors also acknowledge the Fund for Scientific Research-Flanders (FWO) Belgium, the Research Council of the Vrije Universiteit Brussel (OZR), and the University of Antwerp (BOF) for their funding.

\section{References}

[1] M. Majumder, T. K. Gangopadhyay, A. K. Chakraborty, K. Dasgupta, and D. K. Bhattacharya, "Fibre Bragg gratings in structural health monitoring-present status and applications," Sensors and Actuators, A: Physical, vol. 147, no. 1, pp. 150-164, 2008.
[2] A. Othonos, "Fiber Bragg gratings," Review of Scientific Instruments, vol. 68, no. 12, pp. 4309-4341, 1997.

[3] A. D. Kersey, M. A. Davis, H. J. Patrick et al., "Fiber grating sensors," Journal of Lightwave Technology, vol. 15, no. 8, pp. 1442-1463, 1997.

[4] T. Vella, S. Chadderdon, R. Selfridge et al., "Full-spectrum interrogation of fiber bragg gratings at $100 \mathrm{kHz}$ for detection of impact loading," Measurement Science and Technology, vol. 21, no. 9, Article ID 094009, 2010

[5] W. Nakwaski and M. Osinski, "Thermal properties of verticalcavity surface-emitting semiconductor lasers," in Progress in Optics, vol. 38, pp. 165-262, 1998.

[6] T. Mizunami, S. Hirose, T. Yoshinaga, and K.-I. Yamamoto, "Power-stabilized tunable narrow-band source using a VCSEL and an EDFA for FBG sensor interrogation," Measurement Science and Technology, vol. 24, no. 9, Article ID 094017, 2013.

[7] B. Van Hoe, G. Lee, E. Bosman et al., "Ultra small integrated optical fiber sensing system," Sensors, vol. 12, no. 9, pp. 12052 12069, 2012.

[8] Y. H. Huang, C. Lu, P. K. A. Wai, and H. Y. Tam, "Fast FBG sensor interrogation system using vertical cavity surface emitting laser source," in Proceedings of the 14th OptoElectronics and Communications Conference (OECC '09), Hong Kong, July 2009.

[9] Y. H. Huang, T. A. Guo, C. O. Lu, and H.-Y. Tam, "VCSEL-based tilted fiber grating vibration sensing system," IEEE Photonics Technology Letters, vol. 22, no. 16, pp. 1235-1237, 2010.

[10] N. M. M. Maia and J. M. M. e Silva, Theoretical and Experimental Modal Analysis, Research Studies Press, Taunton, UK, 1997.

[11] A. Lamberti, S. Vanlanduit, B. De Pauw, and F. Berghmans, "Influence of fiber bragg grating spectrum degradation on the performance of sensor interrogation algorithms," Sensors, vol. 14, no. 12, pp. 24258-24277, 2014.

[12] A. Lamberti, S. Vanlanduit, B. De Pauw, and F. Berghmans, "A novel fast phase correlation algorithm for peak wavelength detection of fiber Bragg grating sensors," Optics Express, vol. 22, no. 6, pp. 7099-7112, 2014.

[13] K. S. C. Kuang, R. Kenny, M. P. Whelan, W. J. Cantwell, and P. R. Chalker, "Embedded fibre Bragg grating sensors in advanced composite materials," Composites Science and Technology, vol. 61, no. 10, pp. 1379-1387, 2001.

[14] H. Van Der Auweraer, P. Guillaume, P. Verboven, and S. Vanlanduit, "Application of a fast-stabilizing frequency domain parameter estimation method," Journal of Dynamic Systems, Measurement and Control, Transactions of the ASME, vol. 123, no. 4, pp. 651-658, 2001. 


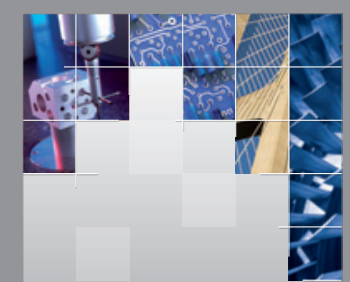

\section{Enfincering}
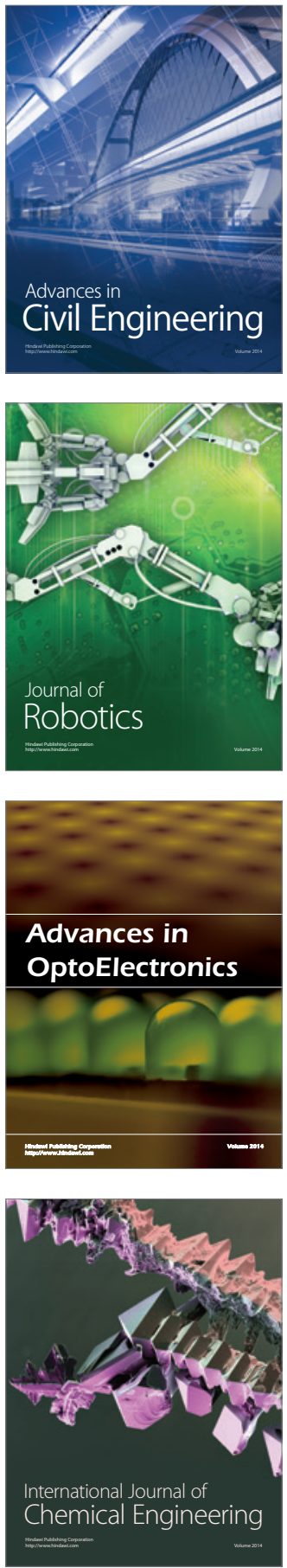

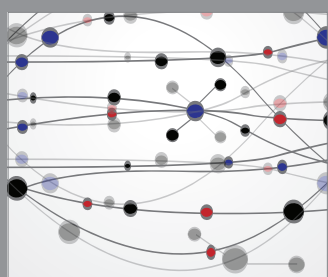

The Scientific World Journal

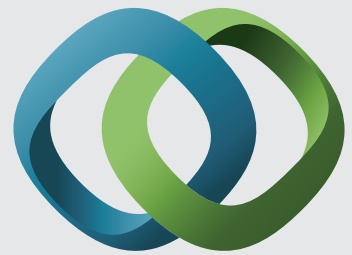

\section{Hindawi}

Submit your manuscripts at

http://www.hindawi.com
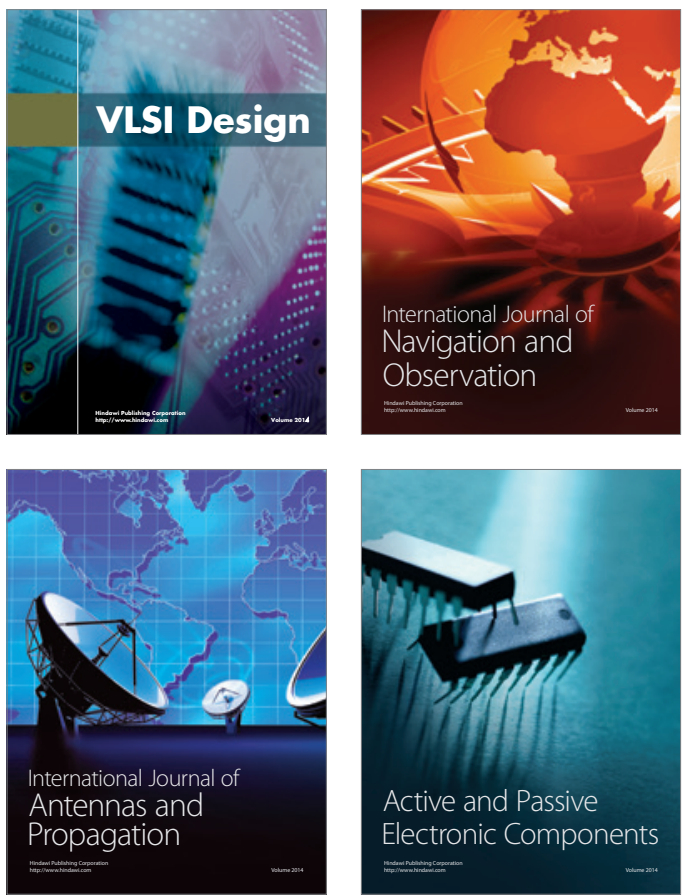
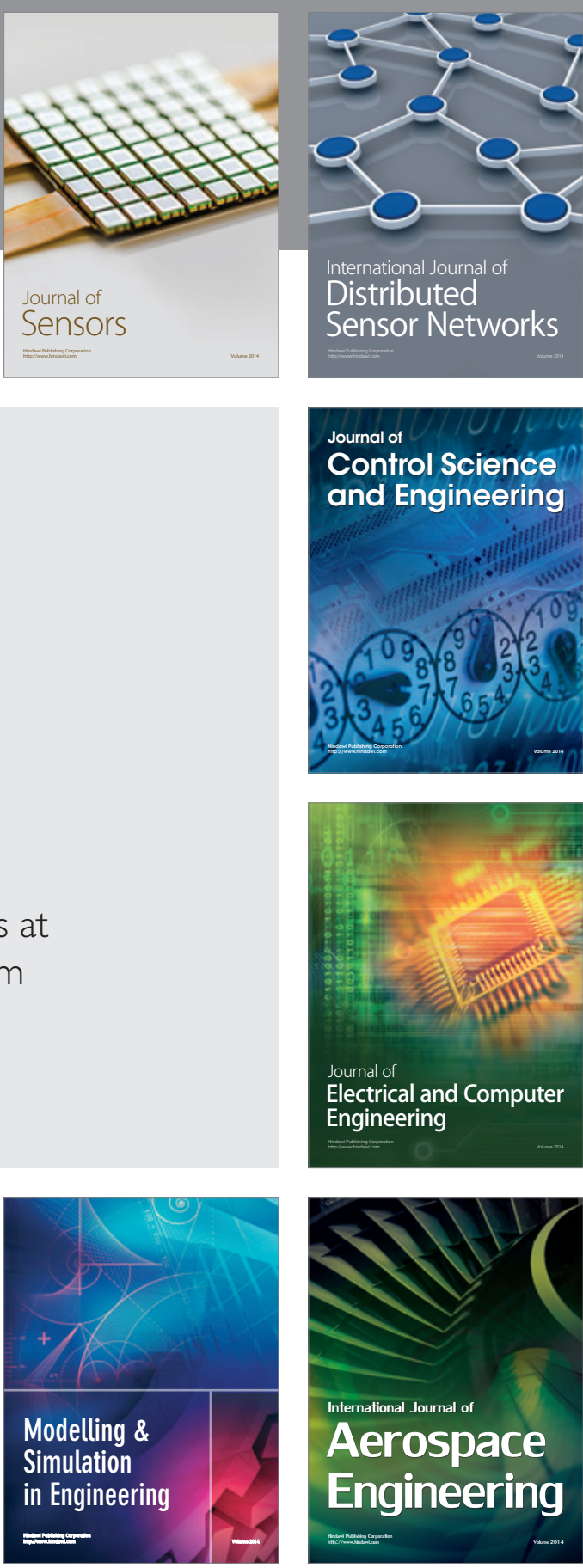

International Journal of

Distributed

Sensor Networks

Journal of

Control Science

and Engineering
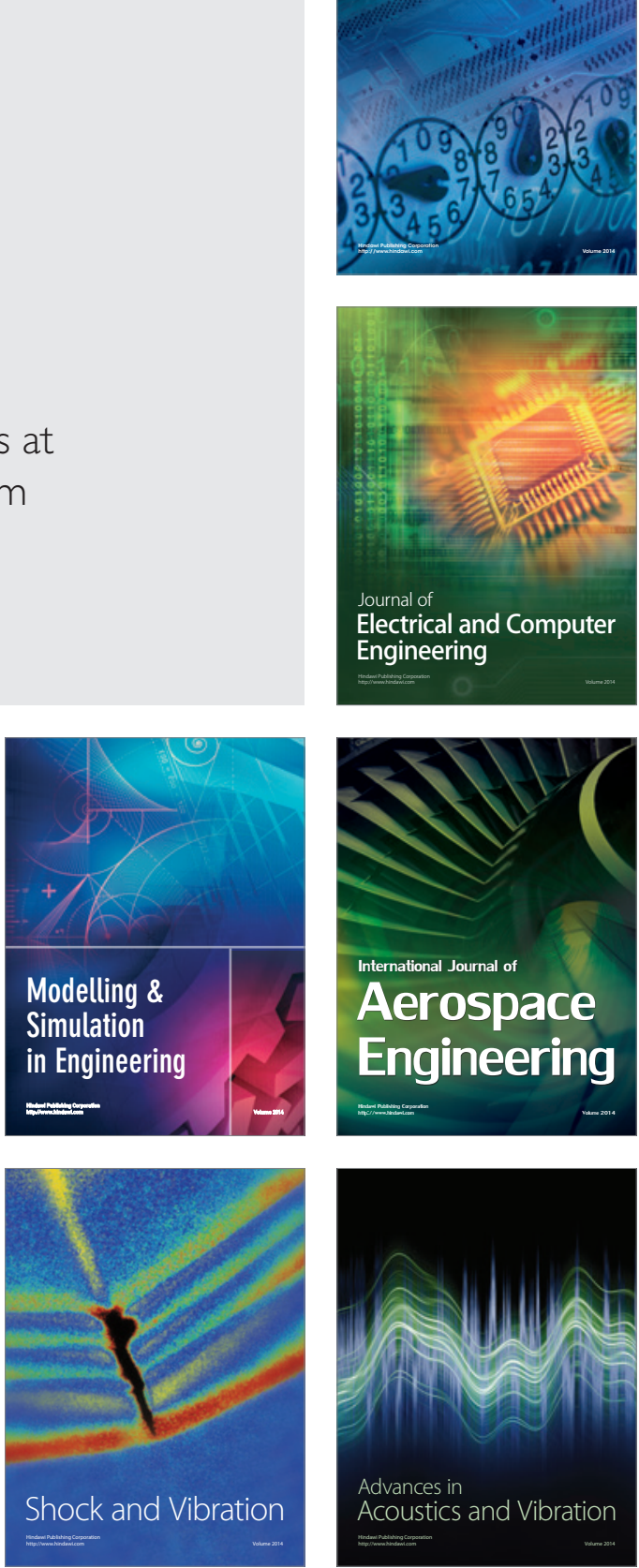